\title{
The Molecular Pathology of Myelodysplastic Syndrome
}

\section{Torsten Haferlach}

MLL Munich Leukemia Laboratory, Munich, Germany

\section{Keywords}

Myelodysplastic syndrome $\cdot$ Cytopenia $\cdot$ Molecular pathology

\begin{abstract}
The diagnosis and classification of myelodysplastic syndromes (MDS) are based on cytomorphology and cytogenetics (WHO classification). Prognosis is best defined by the Revised International Prognostic Scoring System (IPSS-R). In recent years, an increasing number of molecular aberrations have been discovered. They are already included in the classification (e.g., SF3B1) and, more importantly, have emerged as valuable markers for better classification, particularly for defining risk groups. Mutations in genes such as SF3B1 and $\mid D H 1 / 2$ have already had an impact on targeted treatment approaches in MDS.

(c) 2018 S. Karger AG, Basel
\end{abstract}

This review is a short summary of a lecture delivered at the 8th EBMWG International Course and Workshop on Bone Marrow Pathology, Utrecht, The Netherlands, 29 May 2017.

\section{KARGER}

() 2018 S. Karger AG, Basel

E-Mail karger@karger.com

www.karger.com/pat

\section{Introduction}

Myelodysplastic syndrome (MDS) is a clonal disorder characterized by ineffective and dysplastic hematopoiesis. It presents clinically with peripheral blood cytopenias, and can evolve into acute myeloid leukemia (AML).

In addition to cytomorphologic characteristics, the last decades led to enormous gains in knowledge, with respect to the cytogenetic background, and, in recent years, the molecular genetic background of MDS. Already early on, classification systems involved chromosomal information $[1,2]$. Classification systems to implement molecular markers are now just being developed.

By the use of newer sequencing techniques such as next-generation sequencing (NGS), the landscape of molecular pathology in MDS is on the way to being deciphered. Large studies of whole-genome sequencing (WGS) plus RNA sequencing (RNA-Seq) are pending.

\section{The Role of Molecular Changes in Classification}

The already-known molecular changes in MDS, showing correlations to clinical staging systems, such as the Revised International Prognostic Scoring System (IPSS- 


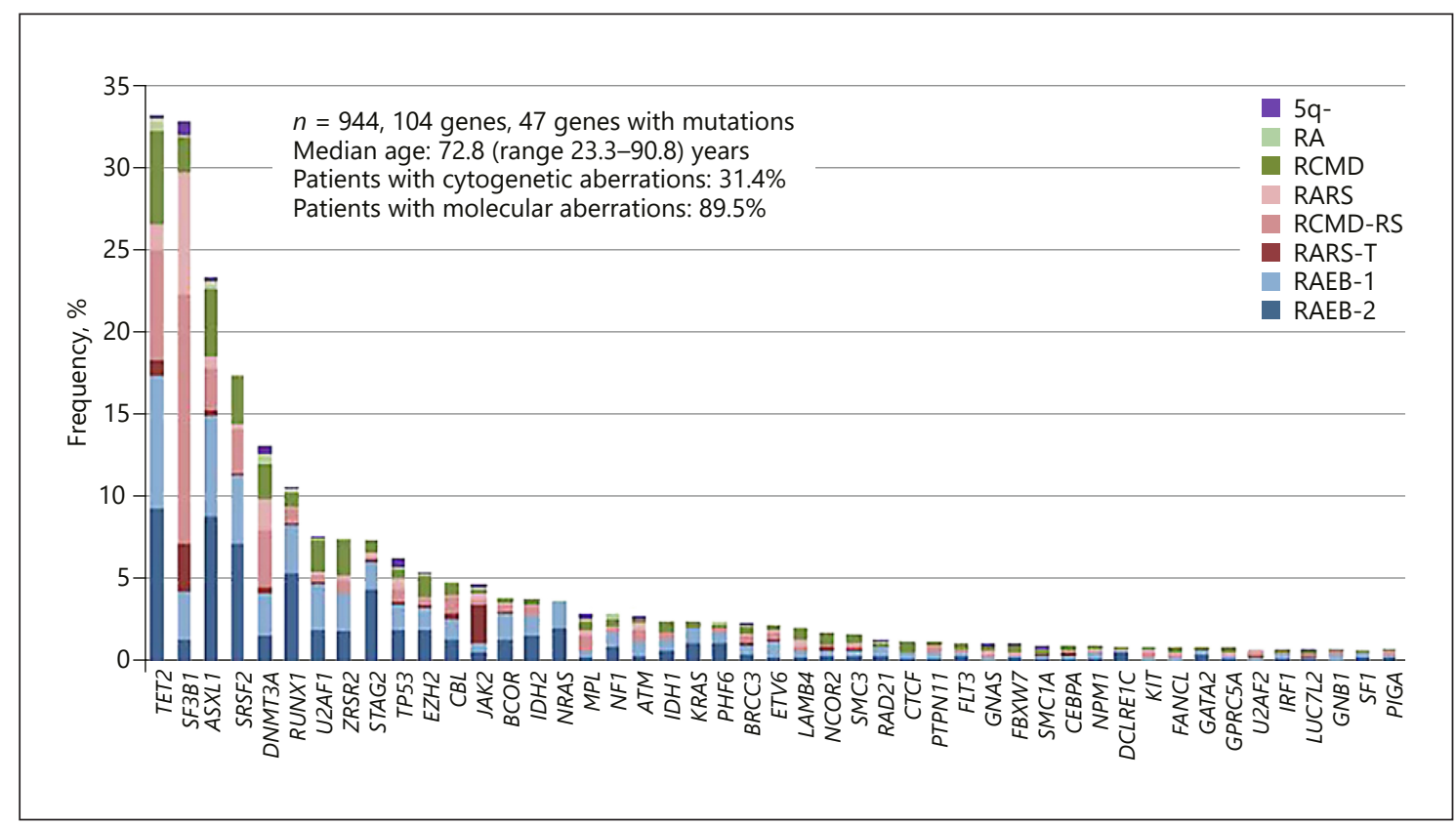

Fig. 1. Incidence of molecular mutations in 944 patients with cytomorphologically proven MDS, after sequencing 104 genes by NGS [7].

R) [2], the WHO classification-based Prognostic Scoring System (WPSS) [3], and the recently updated WHO classification (2017) [4,5], are being increasingly implemented in diagnosis, classification, prognostication, and to guide treatment algorithms.

Although the number of driver genes in MDS is large, as determined by sequencing studies (Fig. 1) [6, 7], these can be organized into a limited number of subtypes, which correspond with the implicated cellular mode of action: RNA-splicing factors, epigenetics regulators, cohesin components, transcription factors, DNA damage response and signal transduction molecules (Fig. 2).

The last European LeukemiaNet recommendations [8] suggested that only mutation analyses of candidate genes should be performed but, today, the state of the art should be a recommendation not only for diagnostic purposes (e.g., SF3B1 according to the WHO [4]), but also for prognostication and clinical management.

Early papers $[9,10]$ clearly demonstrated the incidence of molecular variants in MDS as well as the clinical importance of some of these findings such as the SF3B1 mutation. The latter already translated into a specific treatment option with the drug luspatercept.

\section{The New Role of Molecular Changes in Diagnostics and Prognostication}

Two hallmark papers $[6,7]$ investigated $>1,650$ cases of MDS, all classified according to cytomorphologic criteria and following the WHO 2008 criteria. Cases were investigated in parallel with cytogenetics and a panel of molecular markers (111 or 104 genes, respectively). The incidences of mutated genes are quite similar [11]. Importantly, both papers could distinguish diverse clinical outcomes of patients with MDS, according to the number of driver mutations identified, also for a low IPSS score [6]. In Haferlach et al. [7], the mean number of mutations per patient increased by increasing the blast count. They could also clearly discriminate patients with MDS using a combination of IPSS-R parameters and 14 genes, or, even more advanced, by means of a a 14-gene panel using a bioinformatics model. This clearly demonstrated the extent of the information being gained by molecular investigations into MDS, with $80-90 \%$ of patients showing at least 1 mutation in the investigations $[6,7]$. Thus, molecular data will be included in prognostication. The implementation of molecular information into standard scores, based on dysplasia, percentage of blasts, cytogenetic information, and cytopenias, is on its way. 
Fig. 2. Recurrently mutated genes in MDS organized into 2 biological categories by means of the combined dataset adapted from Papaemmanuil et al. [10], Haferlach et al. [7], and Lindsley (pers. commun. in [24]).

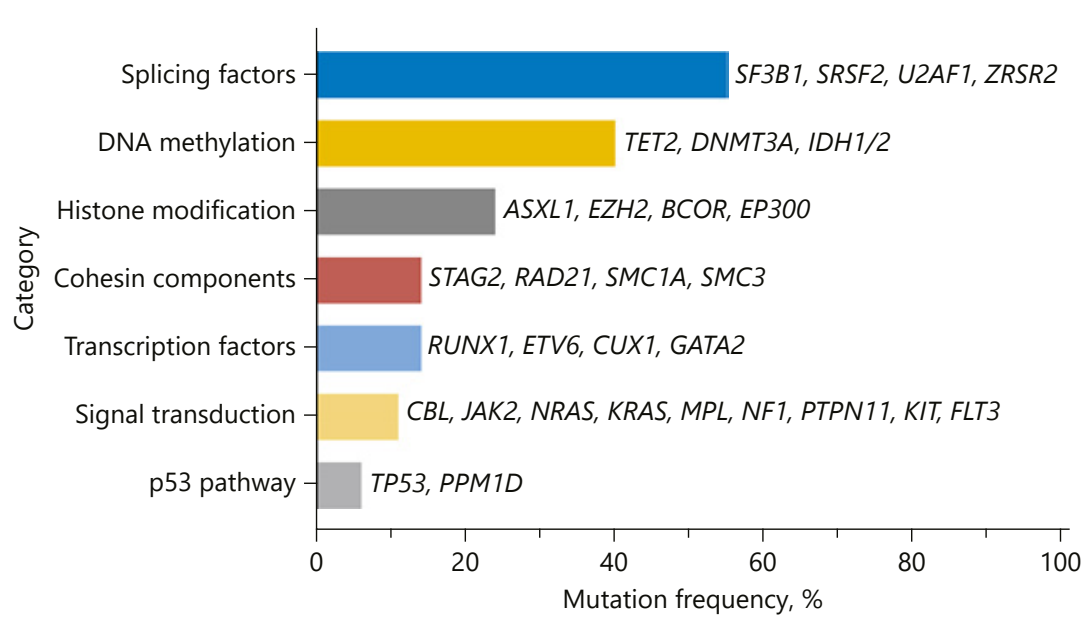

In a subgroup "MDS with isolated 5q-deletion," the recent WHO classification recommends investigating TP53. This can be found in around 20\% of MDS patients with an isolated $5 \mathrm{q}$-deletion and is correlated with inferior outcome. TP53 should also be investigated to determine whether a patient is eligible for treatment with lenalidomide.

Several publications also analyzed MDS and the respective secondary AML, and demonstrated the molecular landscape for MDS and secondary AML at both time points [12], particularly MDS with an isolated 5q-deletion [13].

\section{The Role of Molecular Changes for Treatment Choice}

From the clinical and therapeutic point of view, the options for targeted treatment of MDS are rapidly increasing [14]. Different pathogenic mechanisms, such as cytokine aberrations, abnormal mRNA splicing, telomere dysfunction, abnormal microenvironment, immune system dysregulation as well as abnormal epigenetic regulation, lead to very specific therapeutic options that can be combined in daily routine settings. It seems mandatory, especially within studies, that patients are investigated not only according to cytomorphology and cytogenetics, but also a broad spectrum of molecular markers and their expression to guide treatment. One example is the isocitrate dehydrogenase inhibitors as well as activin antagonists such as luspatercept, immunomodulators like tha- lidomide or lenalidomide, or hypomethylating agents like azacytidine and decitabine.

Some clinical guidelines already suggest the study of MDS on the molecular level at the time point of diagnosis, especially in patients with a low or intermediate risk according to the IPSS or IPSS-R (https://dgho.de, https:// www.onkopedia-guidelines.info/en/onkopedia/guidelines/myelodysplastic-syndromes). The genes to be investigated, especially because of their negative prognostic impact, are TP53, ASXL1, RUNX1, and EZH2.

A controversial issue is whether or not to perform allogeneic transplantation, as the only curative treatment for MDS, based on molecular markers only. In cases of clonal cytopenias of undetermined significance (CCUS), which may be diagnosed many years before dysplasia is recognized, the best clinical approach needs to be discussed and evaluated in prospective studies.

\section{Bioinformatics and Accreditation}

Bioinformatics tools are still the bottleneck for data interpretation. Although a lot of knowledge has already been gained and combined in international networks like the International Working Group for MDS Molecular Prognosis Committee, the routine diagnostic application of molecular studies for MDS needs a lot of software tools and bioinformatics help. This is especially true for variants that have not been described in MDS patients before. They need a lot of bioinformatics, and automated interpretation based on international databases and laboratory in-house data. Functional studies may also be need- 




Fig. 3. Mutations in patients with MDS according to morphology and cytogenetics analysis, and cases with cytopenia but no definite morphological diagnosis (MDS possible, MDS not visible, Unclear, and Reactive). Genes with at least 1 mutation are shown in red, variants of uncertain significance (VUS) in orange, unmutated genes in gray, and not analyzed (N.A.) genes in white [23].

ed, and the final interpretation of all these new genetic findings still needs to be evaluated in the clinical context.

It further should be emphasized that several guidelines have been published for the implementation and accreditation of an NGS workflow and its data interpretation for laboratories [15-18].

As pre-MDS diseases and clinical scenarios are common, e.g., cytopenias, idiopathic cytopenias of undetermined significance (ICUS), CCUS, and clonal hematopoiesis of indeterminate potential (CHIP) [19], these must all be separated from clear-cut MDS. Several recent papers have published their experiences regarding clinical wording $[20,21]$ and clinical use in a routine setting [22, 23]. Malcovati et al. [22] and Baer et al. [23] investigated unclear cytopenias without clear-cut morphological changes and normal karyotype with gene panels (Fig. 3). This further subclassifies patients and lead to a diagnosis. More studies are warranted to define the respective scenarios in a routine setting for the approach for patients with unexplained cytopenias.

\section{The Next Steps}

The increasing need to study MDS patients at diagnosis, or, at the latest, in progression, will only be deliverable by the use of NGS. So far, panel testing is the method of choice. The laboratory set-up will be able to produce informative data within 1-2 weeks. This turnaround time is acceptable for any further decision, from the clinical point of view, about the MDS patient.

With all of this in mind, one can speculate about the diagnostic and therapeutic advances that will take place in MDS and hematology in the next 5 years. For sure, the amount of data will increase dramatically as will the development of new treatment options. More precise approaches, that follow a comprehensive diagnostic workup and lead to precision medicine, will be implemented in the treatment of MDS patients. Three important aspects will precipitate this: (1) the technique to define the respective patient's disease and biology will be available, (2) the assays will be delivered in a routine setting, and (3) the health care system will offer reimbursement. This will 
automatically foster the development of new treatments, bearing in mind that finding SF3B1 mutations in 2011 to developing the first drugs for humans took only 5 years.

With respect to the implementation of RNA-Seq and WGS, or whole-exome sequencing, the value of these novel modalities in clinical practice has still to be proven in large patient cohorts before they can be considered useful for clinical use.

Instruments and assays, investigating a large number of samples by means of panels, exomes, and even WGS combined with RNA-Seq, will develop quickly. This will dramatically change the hematologic diagnostic approach. In parallel, turnaround times will become more acceptable and bioinformatics interpretation of data will be more automized. Today's workflows can even sequence genomes with a coverage of $100 \times$ within a week. In 5 years from now, this may be possible at a cost of USD 300 . Techniques like liquid biopsy are already being tested, not only for solid tumors but also in hematologic patients. As tumor and leukemia cells change their biological repertoire over the course of the disease and especially during treatment, several additional time points to analyze and describe the respective cancer, and adopt treatments accordingly, will be normal for patients with MDS in the next 5 years.

It is our duty to implement all available options for diagnostic approach and testing in clinical trials, exploring all possible treatments in order to offer the best support to patients. In MDS, this will open completely new paths for the care and cure of patients.

In conclusion, genomics in MDS today is needed to improve diagnosis, specify classification and define prognosis, allow individualized treatment, and even offer strategies for detecting minimal residual disease. This will all lead to a reduction in treatment toxicity and, hopefully, even increase the rate of cure. Furthermore, the development of new drugs will be fostered by comprehensive molecular characterization for patients with MDS.

\section{Disclosure Statement}

There were no conflicts of interest.

\section{References}

1 Greenberg P, Cox C, LeBeau MM, Fenaux P, Morel P, Sanz G, et al: International Scoring System for evaluating prognosis in myelodysplastic syndromes. Blood 1997;89:2079-2088.

-2 Greenberg PL, Tuechler H, Schanz J, Sanz G, Garcia-Manero G, Sole F, et al: Revised International Prognostic Scoring System (IPSS-R) for myelodysplastic syndromes. Blood 2012; 120:2454-2465.

- 3 della Porta MG, Tuechler H, Malcovati L, Schanz J, Sanz G, Garcia-Manero G, et al: Validation of WHO classification-based Prognostic Scoring System (WPSS) for myelodysplastic syndromes and comparison with the revised International Prognostic Scoring System (IPSS-R). A study of the International Working Group for Prognosis in Myelodysplasia (IWG-PM). Leukemia 2015;29:15021513.

4 WHO Classification of Tumours of Haematopoietic and Lymphoid Tissues, ed 5. Lyon, IARC, 2017.

5 Arber DA, Orazi A, Hasserjian R, Thiele J, Borowitz MJ, Le Beau MM, et al: The 2016 revision to the World Health Organization classification of myeloid neoplasms and acute leukemia. Blood 2016;127:2391-2405.
6 Papaemmanuil E, Gerstung M, Malcovati L, Tauro S, Gundem G, Van Loo P, et al: Clinical and biological implications of driver mutations in myelodysplastic syndromes. Blood 2013;122:3616-3627.

7 Haferlach T, Nagata Y, Grossmann V, Okuno Y, Bacher U, Nagae G, et al: Landscape of genetic lesions in 944 patients with myelodysplastic syndromes. Leukemia 2014;28:241247.

8 Malcovati L, Hellstrom-Lindberg E, Bowen D, Ades L, Cermak J, Del Canizo MC, et al: Diagnosis and treatment of primary myelodysplastic syndromes in adults: recommendations from the European LeukemiaNet. Blood 2013;122:2943-2964.

-9 Bejar R, Stevenson K, Abdel-Wahab O, Galili N, Nilsson B, Garcia-Manero G, et al: Clinical effect of point mutations in myelodysplastic syndromes. N Engl J Med 2011;364:24962506.

10 Papaemmanuil E, Cazzola M, Boultwood J, Malcovati L, Vyas P, Bowen D, et al: Somatic SF3B1 mutation in myelodysplasia with ring sideroblasts. N Engl J Med 2011;365:13841395.
11 Rose D, Kohlmann A, Nagata Y, Ogawa S, Haferlach C, Kern W, et al: A robust molecular pattern for myelodysplastic syndromes in two independent cohorts investigated by next-generation sequencing can be revealed by comparative bioinformatic analyses. $\mathrm{Br} \mathrm{J}$ Haematol 2014;167:278-281.

-12 Meggendorfer M, de Albuquerque A, Nadarajah N, Alpermann T, Kern W, Steuer K, et al: Karyotype evolution and acquisition of FLT3 or RAS pathway alterations drive progression of myelodysplastic syndrome to acute myeloid leukemia. Haematologica 2015;100:e487-e490.

13 Meggendorfer M, Haferlach C, Kern W, Haferlach T: Molecular analysis of myelodysplastic syndrome with isolated deletion of the long arm of chromosome 5 reveals a specific spectrum of molecular mutations with prognostic impact: a study on 123 patients and 27 genes. Haematologica 2017;102:1502-1510.

14 Zahid MF, Patnaik MM, Gangat N, Hashmi SK, Rizzieri DA: Insight into the molecular pathophysiology of myelodysplastic syndromes: targets for novel therapy. Eur J Haematol 2016;97:313-320.

15 Matthijs G, Souche E, Alders M, Corveleyn A, Eck S, Feenstra I, et al: Guidelines for diagnostic next-generation sequencing. Eur J Hum Genet 2016;24:2-5. 
16 Richards S, Aziz N, Bale S, Bick D, Das S, Gastier-Foster J, et al: Standards and guidelines for the interpretation of sequence variants: a joint consensus recommendation of the American College of Medical Genetics and Genomics and the Association for Molecular Pathology. Genet Med 2015;17:405424.

$\checkmark 17$ Rehm HL, Bale SJ, Bayrak-Toydemir P, Berg JS, Brown KK, Deignan JL, et al: ACMG clinical laboratory standards for next-generation sequencing. Genet Med 2013;15:733-747.

18 Richards CS, Bale S, Bellissimo DB, Das S, Grody WW, Hegde MR, et al: ACMG recommendations for standards for interpretation and reporting of sequence variations: revisions 2007. Genet Med 2008;10:294-300.

19 Jaiswal S, Fontanillas P, Flannick J, Manning A, Grauman PV, Mar BG, et al: Age-related clonal hematopoiesis associated with adverse outcomes. N Engl J Med 2014;371:24882498.

20 Valent P, Orazi A, Steensma DP, Ebert BL, Haase D, Malcovati L, et al: Proposed minimal diagnostic criteria for myelodysplastic syndromes (MDS) and potential pre-MDS conditions. Oncotarget 2017;8:73483-73500.

21 Steensma DP, Bejar R, Jaiswal S, Lindsley RC, Sekeres MA, Hasserjian RP, Ebert BL: Clonal hematopoiesis of indeterminate potential and its distinction from myelodysplastic syndromes. Blood 2015;126:9-16.

22 Malcovati L, Galli A, Travaglino E, Ambaglio I, Rizzo E, Molteni E, et al: Clinical significance of somatic mutation in unexplained blood cytopenia. Blood 2017;129:3371-3378.

-23 Baer C, Pohlkamp C, Haferlach C, Kern W, Haferlach T: Molecular patterns in cytopenia patients with our without evidence of myeloid neoplasm - a comparison of 756 cases. Leukemia 2018, DOI: 10.1038/s41375-0180119-8.

24 Kennedy JA, Ebert BL: Clinical implications of genetic mutations in myelodysplastic syndrome. J Clin Oncol 2017;35:968-974. 